\title{
Identification of markers for migrasome detection
}

\author{
Xiaoxin Zhao ${ }^{1}$, Yaxin Lei ${ }^{1}$, Jiajia Zheng ${ }^{2}$, Junya Peng ${ }^{3}$, Ying Li ${ }^{4}$, Li Yu ${ }^{1}$ and Yang Chen ${ }^{5}$
}

\section{Dear Editor,}

The migrasome is a newly discovered cellular organelle in migrating cells, first described in $2015^{1,2}$. Migrasomes have been proposed to mediate cell-cell communications and may exert physiological and pathological effects. In zebrafish embryos, generation of migrasomes has been observed during gastrulation, and migrasomes have been shown to be essential for organ morphogenesis during embryonic development (Personal communication). Although migrasome-like structures have been detected by transmission electron microscopy (TEM) in various organs including eye, lung and intestine, definitive evidence for the presence of migrasomes in vivo in mammals is still lacking. Exosomes are defined as $30-100 \mathrm{~nm}$ extracellular vesicles derived from the multivesicular body and are secreted by a variety of cell types. Exosomes have attracted great attention in clinical studies since they were detected in solid tissues, such as cartilage $^{3}$, and in biological fluids, such as blood ${ }^{4}$. One of the most commonly used methods for detection of extracellular vesicles (EVs) is a biochemical approach using known marker proteins. This strategy allows researchers to identify EVs in complex biological samples such as body fluids and tissues, and thus provides great flexibility for detection of EVs in vivo. So far, there is no satisfactory marker for biochemical detection of migrasomes. TSPAN4 and integrin are known to be enriched on migrasomes ${ }^{1,5}$. However, both proteins are also present on exosomes ${ }^{6}$, making it difficult to distinguish the two structures biochemically based only on detection of these proteins. Imaging is the only approach for confirming the presence of migrasomes, but it is not

\footnotetext{
Correspondence: Li Yu (liyulab@mail.tsinghua.edu.cn) or

Yang Chen (chenyang1816185048@bjmu.edu.cn)

${ }^{1}$ The State Key Laboratory of Membrane Biology, Tsinghua University-Peking

University Joint Center for Life Sciences, School of Life Sciences, Tsinghua University, Beijing 100084, China

${ }^{2}$ Department of Laboratory Medicine, Peking University Third Hospital, Beijing 100191, China

Full list of author information is available at the end of the article.
}

compatible with most clinical samples. Thus, there is an urgent need to identify migrasome-specific markers.

Our strategy to identify migrasome-specific protein markers is to select candidate proteins based on quantitative mass spectrometry analysis on purified migrasomes and exosomes, followed by verification using cell biology and biochemistry approaches. To generate migrasomes, we used NRK cells stably expressing TSPAN4-GFP (Supplementary Fig. S1a). The crude migrasome extract was resuspended in $19 \%$ density medium and subjected to density gradient centrifugation. Migrasomes floated to an interval between 10-15\% (Supplementary Fig. S1b, fractions 4-6). Western blotting analysis confirmed the presence of the migrasome markers TSPAN4 (labeled by GFP) and integrin $\alpha 5$ in fractions 4-6 (Supplementary Fig. S1c). Exosomes were purified using conventional approaches and verified by TEM and western blotting analysis ${ }^{7}$ (Supplementary Fig. S1d-f). The structures of migrasomes and exosomes were compared by TEM and cryo-EM analyses (Fig. 1a, b). Migrasomes are phospholipid bilayer organelles with diameters of $\sim 500 \mathrm{~nm}$ and some purified migrasomes were still attached to retraction fibers. Exosomes are $\sim 100 \mathrm{~nm}$ in diameter. Thus, the two structures are different in morphology and size (Supplementary Fig. S1g).

We employed tandem mass tag (TMT) labeling followed by quantitative mass spectrometry to identify proteins enriched on migrasomes. Purified migrasomes and cell bodies were labeled with different tags of different mass. The samples were then combined and analyzed to determine the proteins enrichment on migrasomes compared to cell bodies (Supplementary Fig. S2a). Three independent migrasome preparations were analyzed by TMT-based quantitative mass spectrometry. Of the total protein hits, 4737 (54.1\%) were reproducibly detected. Of these, we selected 577 proteins which met the criteria that protein score in SEQUEST software $>2$ and migrasometo-cell-body ratio $>1.5$ in each of the three data sets 
a

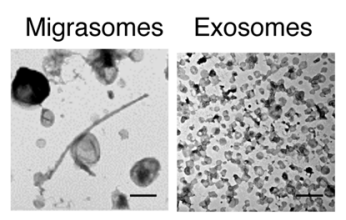

b

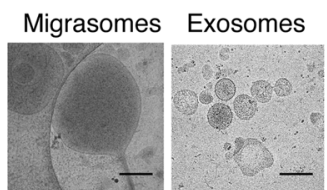

f

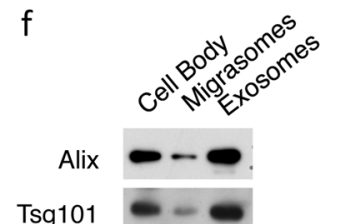

Tsg101

Calnexin

Sec61a

GM130

Tim23

NDST1

PIGK

$\mathrm{CPQ}$

EOGT

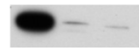

-
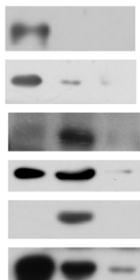$$
\text { jo }
$$

J
Negative
staining

Ultra-thin
section

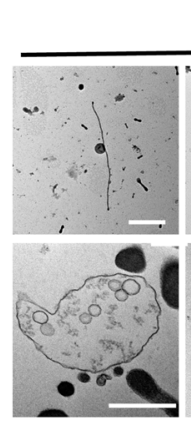

c

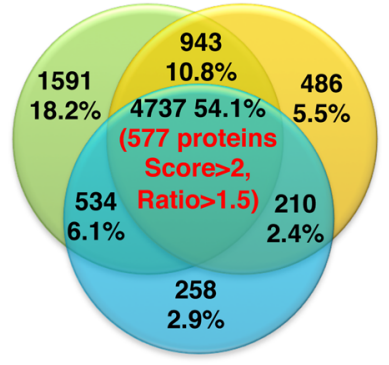

g

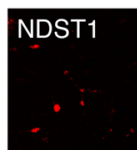

PIGK

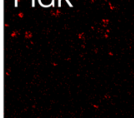

$\mathrm{CPQ}$

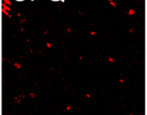

EOGT

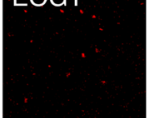

TS
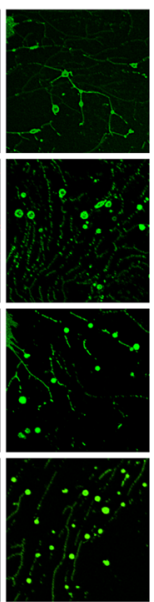

.

Serum
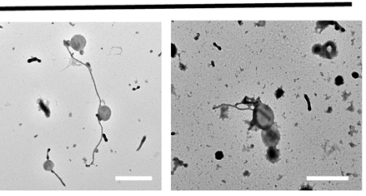

NRK

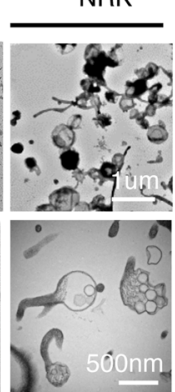

d

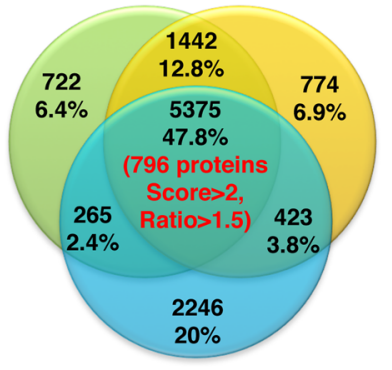

e

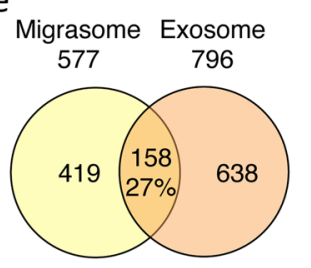

$\mathrm{h}$

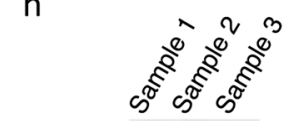

Integrin a5

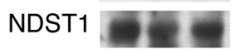

EOGT

PIGK

CPQ

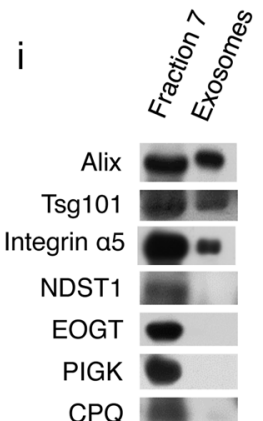

Fig. 1 Identification of migrasome-specific markers based on quantitative mass spectrometry. a Representative TEM images of negatively stained samples of migrasomes and exosomes purified from NRK cells overexpressing TSPAN4-GFP. Scale bar, $500 \mathrm{~nm}$. b Representative cryo-EM images of migrasomes and exosomes purified from NRK cells overexpressing TSPAN4-GFP. Scale bar, $200 \mathrm{~nm}$. c Overlap of proteins from migrasomes identified in three independent quantitative mass spectrum analyses. The central overlapping area shows the 4737 proteins that were reproducibly detected in all three analyses. 577 of these proteins have score $>2$ and ratio $>1.5$. $\mathbf{d}$ Overlap of proteins from exosomes identified in three independent quantitative mass spectrum analyses. The central overlapping area shows the 5375 proteins that were reproducibly detected in all three analyses.796 of these proteins have score $>2$ and ratio $>1.5$. e Venn diagram showing the number of proteins enriched on migrasomes and exosomes with score $>2$ and ratio $>1.5$ in all three repeats. The total number of proteins is shown above the diagram, and the unique proteins and shared proteins are shown in the circles. $\mathbf{f}$ Samples from cell bodies and purified migrasomes and exosomes were analyzed by western blotting using antibodies against Alix, Tsg101, Calnexin, Sec61a, GM130 and Tim23, and identified migrasome-specific marker NDST1, PIGK, CPQ and EOGT. g NRK cells stably expressing TSPAN4-GFP were cultured on a fibronectin-coated surface. NDST1, PIGK, CPQ and EOGT were detected by immuno-

fluorescence using the corresponding antibodies. Scale bar: $5 \mu \mathrm{m}$. $\mathbf{h}$ Serum samples from three different individuals ( $1 \mathrm{ml} / \mathrm{Sample})$ were subjected to centrifugation at $20000 \times \mathrm{g}$. The pellet was analyzed by western blot using antibodies against indicated proteins. $\mathbf{i}$ Fraction 7 from Supplementary Fig. $4 \mathrm{~B}$ and exosomes purified from serum samples were analyzed by antibodies against Alix, Tsg101, Integrin $\beta 1$, NDST1, EOGT, PIGK and CPQ. $\mathbf{j}$ Representative TEM images of negatively stained samples and embedded ultra-thin sections of migrasomes purified from human serum and from NRK cells stably expressing TSPAN4-GFP. Scale bars are indicated in the figure 
(Fig. 1c). The enrichment of the proteins on migrasomes is 1.5 times compared to cell bodies. GO classification showed that the genes encoding these 577 proteins are enriched for GO terms includingcell migration, cellsubstrate adhesion, lipid catabolicprocesses as well as protein glycosylation and glycoprotein metabolic processes (Supplementary Fig. S2b). We acquired exosome proteomic data using the same approach and selected a list of 796 proteins enriched on exosomes using the same criteria (Fig. 1d). The comparison of migrasome and exosome proteomics showed that the two structures shared only 158 (27\%) proteins (Fig. 1e).

Base on the MS data, we screened for proteins enriched only on migrasomes using purified migrasomes and exosomes samples. We first confirmed that purified migrasomes and exosomes contain known protein markers without contamination of other cellular organelles (Fig. 1f). Western blotting results showed that four proteins, NDST1 (bifunctionalheparan sulfate $\mathrm{N}$-deacetylase/ $\mathrm{N}$-sulfotransferase 1 ), PIGK (phosphatidylinositol glycan anchor biosynthesis, class K), CPQ (carboxypeptidase Q) and EOGT (EGF domainspecific O-linked $\mathrm{N}$-acetylglucosaminetransferase), were enriched in migrasomes, but were absent or barely detectable in exosomes (Fig. 1f, Supplementary Fig. S3a). We observed that mcherry-labeled four proteins localized on migrasomes with live cell imaging (Supplementary Fig. S3b). We also stained cells with specific antibodies against these proteins, and found that all of them are indeed localized in migrasomes (Fig. 1g).

With these markers available, we next set out to detect migrasomes in human serum. We first took $1 \mathrm{ml}$ serum samples each from three individuals and found that all four proteins and integrin $\alpha 5$ were detected (Fig. 1h), indicating that migrasomes may be present in serum. We further fractionated human serum using a protocol similar to that for isolating migrasomes from cultured cells (Supplementary Fig. S4a), and found that fraction 7 contained all 4 identified migrasome markers and integrin $\alpha 5$ (Supplementary Fig. S4b), which strongly suggests that migrasomes are present in serum. Next, we compared fraction 7 and purified exosome fraction from serum samples and found that all four migrasome markers were exclusively present in fraction 7 but not in exosomes, which is consistent with the marker specificity in detecting migrasome obtained from cultured cells (Fig. 1i).

To directly verify the presence of migrasomes in serum, we subjected the sample from fraction 7 to negative staining and TEM revealed vesicles attached to tubules, which are characteristic morphological features of migrasomes (Fig. $1 j$ ). The diameter of the structures purified from serum was very similar to migrasomes purified from NRK cells (Supplementary Fig. S4c). We also embedded the sample from fraction 7 and subjected it to ultra-thin section TEM. This uncovered another characteristic morphological feature of migrasomes, which is the presence of small luminal vesicles within larger vesicles (Fig. 1j). From these data, we conclude that migrasomes are present in human serum.

In this manuscript, we compared the protein composition of migrasomes and exosomes by quantitative MS analysis. Using this information, we successfully identified four marker proteins for migrasomes, which are not present in exosomes. Identification of these migrasomespecific markers enabled us to detect the presence of migrasomes biochemically. Our study identified a set of markers for biochemical detection of migrasomes that is compatible with clinical samples, and will potentially be important in future disease-related studies. Moreover, it may open avenues for translational studies of migrasomes in diagnostic, prognostic and therapeutic applications.

Using this approach, we surprisingly found that migrasomes are present in human serum. The presence of migrasomes immediately raises several questions. Where are migrasomes generated, and by which cell type? How do migrasomes enter the circulatory system? Where do migrasomes go and what is their function in serum? Are serum migrasomes related to certain diseases, and can they be used as a diagnostic tool? Future investigations are needed to address these questions.

\begin{abstract}
Acknowledgements
We are grateful to the Department of Laboratory Medicine, Peking University 3rd Hospital, for the blood samples. We thank the protein chemistry facility at the Center for Biomedical Analysis of Tsinghua University for the quantitative mass spectrum analysis. We thank the Cell Facility in the Center of Biomedical Analysis of Tsinghua University for help with electron microscopy. We thank the National Center for Protein Science (Beijing) Tsinghua Cryo-EM facility for providing technical support. We thank the Tsinghua Cell Biology Core Facility for cell imaging. This research was supported by the Ministry of Science and Technology of the People's Republic of China (2018YFA0507102 to Yang Chen, 2016YFA0500202 and 2017 YFA0503404 to Li Yu), and the National Natural Science Foundation of China (91754108 and 31671395 to Yang Chen, 31430053 and 31321003 to Li Yu).
\end{abstract}

\section{Author details}

${ }^{1}$ The State Key Laboratory of Membrane Biology, Tsinghua University-Peking University Joint Center for Life Sciences, School of Life Sciences, Tsinghua University, Beijing 100084, China. ${ }^{2}$ Department of Laboratory Medicine, Peking University Third Hospital, Beijing 100191, China. ${ }^{3}$ Medical Research Center, Peking Union Medical College Hospital, Beijing 100043, China. ${ }^{4}$ Tsinghua University-Peking University Joint Center for Life Sciences, technology center for protein sciences, School of Life Sciences, Tsinghua University, Beijing 100084, China. ${ }^{5}$ Center for Precision Medicine Multi-Omics Research, Peking University Health Science Center, Peking University, Beijing 100191, China

\section{Authors' contributions}

L.Y. and Y.C. designed the study. Y.C., X.Z., Y.L., J.Z., J.P., Y.L. performed experiments and data analysis. Y.C. and L.Y. wrote the manuscript.

Conflict of interest

The authors declare that they have no conflict of interest.

\section{Publisher's note}

Springer Nature remains neutral with regard to jurisdictional claims in published maps and institutional affiliations. 
Supplementary Information accompanies the paper at (https://doi.org/ 10.1038/s41421-019-0093-y).

Received: 28 September 2018 Revised: 15 March 2019 Accepted: 15 March 2019

Published online: 21 May 2021

\section{References}

1. Ma, L. et al. Discovery of the migrasome, an organelle mediating release of cytoplasmic contents during cell migration. Cell Res. 25, 24-38 (2015).
2. da Rocha-Azevedo, B. \& Schmid, S. L. Migrasomes: a new organelle of migrating cells. Cell Res. 25, 1-2 (2015).

3. Anderson, H. C. Vesicles associated with calcification in the matrix of epiphyseal cartilage. J Cell Biol. 41, 59-72 (1969).

4. Crawford, N. The presence of contractile proteins in platelet microparticles isolated from human and animal platelet-free plasma. Br J Haemato/ 21, 53-69 (1971)

5. Wu, D. et al. Pairing of integrins with ECM proteins determines migrasome formation. Cell Res. 27, 1397-1400 (2017).

6. Thery, C., Zitvogel, L. \& Amigorena, S. Exosomes: composition, biogenesis and function. Nat Rev Immunol. 2, 569-579 (2002).

7. Coumans, F. A. W. et al. Methodological Guidelines to Study Extracellular Vesicles. Circul Res. 120, 1632-1648 (2017). 